\title{
Preoperative Polyethylene Glycol versus a Single Enema in Elective Bowel Surgery
}

\author{
A.A.F.A. Veenhof C. Sietses G.F. Giannakopoulos D.L. van der Peet \\ M.A. Cuesta
}

Department of Surgery, VU University Medical Center, Amsterdam, The Netherlands

\section{Key Words}

Colorectal surgery • Bowel preparation, colorectal surgery •

Polyethylene glycol $\cdot$ Single enema

\begin{abstract}
Background: At the beginning of 2004, a large Dutch randomized study comparing mechanical bowel preparation with a single preoperative enema reported no differences in anastomotic leakage rates, septic complications, or mortality. On the basis of this study, this institution abandoned the routine practice of bowel preparation with polyethylene glycol and introduced a single preoperative enema. Methods: A consecutive series of 78 patients undergoing elective colorectal surgery with mechanical bowel preparation using polyethylene glycol (PG group) was compared to a consecutive series of 71 patients with a single preoperative enema (SE group). The PG group was operated between June 2003 and December 2004, the SE group between January 2005 and January 2006. Mortality, anastomotic leakage, and infectious and noninfectious complications were compared. Results: Mortality, anastomotic leakage, frequency of reoperations, and hospital stay were comparable for both groups. The overall postoperative morbidity was significantly higher in the SE group (26 vs. 9, $p=0.003$ ). Specifically, the incidence of wound infections was significantly higher in the SE group (7 vs. $1, p=0.041$ ). Furthermore, a trend was observed in the incidence of intra-abdominal infections which was higher in
\end{abstract}

the SE group (8 vs. $2, p=0.070$ ). Also a trend toward more extra-abdominal infectious complications was seen in the $\mathrm{SE}$ group (11 vs. $4, p=0.086$ ). Conclusions: Based on both the conflicting data currently available from different randomized studies and our results, the preoperative bowel preparation protocol in our institution has been changed. All leftsided colonic resections, in patients who can tolerate mechanical bowel preparation, are prepared with polyethylene glycol.

Copyright $\odot 2007$ S. Karger AG, Basel

\section{Introduction}

At the beginning of 2004, results from a large Dutch randomized study comparing mechanical bowel preparation to a single enema in elective colorectal surgery were presented at the Annual Dutch Surgical Congress [1]. In total, 1,433 patients were included in this multicenter trial. No differences were seen in the percentage of anastomotic leakage, septic complications, or mortality. It was concluded that mechanical bowel preparation using polyethylene glycol did not reduce the incidence of postoperative complications and could, therefore, be abandoned. Furthermore, in 2004 a review of various smaller randomized studies was published in the Cochrane Database [2]. The conclusion of this review confirmed the Dutch data and even suggested that bowel

\section{KARGER \\ Fax +4161306 1234 E-Mail karger@karger.ch} www.karger.com (c) 2007 S. Karger AG, Basel 0253-4886/07/0241-0054\$23.50/0

Accessible online at:

www.karger.com/dsu
Miguel A. Cuesta

Department of Surgery, Vrije Universiteit Medical Center

PO Box 7057

NL-1007 MB Amsterdam (The Netherlands)

Tel. +31 20444 4781, Fax +31 20444 4511, E-Mail ma.cuesta@vumc.nl 
Table 1. Patient characteristics

\begin{tabular}{lclc}
\hline & $\begin{array}{l}\text { SE group } \\
(\mathrm{n}=78)\end{array}$ & $\begin{array}{l}\text { PG group } \\
(\mathrm{n}=71)\end{array}$ & $\mathrm{p}$ \\
\hline Gender & & & \\
$\quad$ Male & $33(42 \%)$ & $28(39 \%)$ & 0.741 \\
$\quad$ Female & $45(58 \%)$ & $43(61 \%)$ & \\
Median age, years (interquartile range) & $59(43-68)$ & $64(51-75)$ & 0.091 \\
Median body mass index (interquartile range) & $23.9(21.4-27.2)$ & $24(22.3-26.8)$ & 0.795 \\
\hline
\end{tabular}

Table 2. Type of operation

\begin{tabular}{lccc}
\hline & $\begin{array}{l}\text { SE group } \\
(\mathrm{n}=78)\end{array}$ & $\begin{array}{c}\text { PG group } \\
(\mathrm{n}=71)\end{array}$ & $\mathrm{p}$ \\
\hline Operation & & & 0.810 \\
$\quad$ Abdominoperineal resection & $4(5 \%)$ & $3(4 \%)$ & \\
Anterior resection & $6(8 \%)$ & $10(14 \%)$ & \\
Left colectomy & $4(5 \%)$ & $2(3 \%)$ & \\
Right colectomy & $12(15 \%)$ & $13(18 \%)$ & \\
Subtotal colectomy & $8(10 \%)$ & $7(10 \%)$ & \\
Ileocecal resection & $15(19 \%)$ & $11(15 \%)$ & \\
Sigmoidal resection & $28(36 \%)$ & $22(31 \%)$ & \\
$\quad$ Transverse colon resection & $1(1 \%)$ & $3(4 \%)$ & \\
Median operation time, min (interquartile range) & $160(110-226)$ & $140(120-190)$ & 0.291 \\
Median blood loss, ml (interquartile range) & $200(25-500)$ & $300(150-500)$ & 0.171 \\
\hline & & & \\
\hline
\end{tabular}

preparation might lead to more anastomotic leakages, and, therefore, the procedure should be omitted.

The VU University Medical Center, changed its protocol on the basis of these data, substituting mechanical bowel preparation using polyethylene glycol for a single preoperative enema. These changes were implemented in January 2005 with a planned evaluation after 1 year.

\section{Patients and Methods}

\section{Patients}

A consecutive series of 78 patients undergoing elective colorectal surgery with preoperative bowel preparation using a single enema (SE group) was compared to a consecutive series of $71 \mathrm{pa-}$ tients undergoing elective colorectal surgery with preoperative bowel preparation using polyethylene glycol (PG group). The single enema consisted of a small volume of Phospho-Soda ${ }^{\circledR}$ administered in the hospital. The polyethylene glycol was also administered in the hospital. The group who received a single preoperative enema was operated on between January 2005 and January 2006. The group who received preoperative polyethylene glycol was operated on between June 2003 and December 2004. Only patients undergoing elective colorectal surgery were included. The patients received standard prophylactic antibiotics, as described in hospital protocols.

Polyethylene Glycol versus Single Enema
Data Collection

Patient data: gender, age, and body mass index. Perioperative data: operating time and blood loss. Postoperative complications: (1) mortality; (2) intra-abdominal infections such as postoperative infections at the abdominal cavity, localized (abscess) or not, including infectious anastomotic leakage; (3) reoperations and reoperations due to anastomotic leakage; (4) extra-abdominal infections, including wound infections, and (5) noninfectious complications.

\section{Statistics}

Statistical analysis was performed using SPSS for Windows (version 11.5; SPSS, Chicago, Ill., USA). Median values and interquartile ranges were calculated and subsequently depicted when appropriate. Mann-Whitney U test, chi-squared test, or Fisher's exact test were applied when appropriate for group comparisons. Significance was set at $\mathrm{p}<0.05$.

\section{Results}

The patient characteristics are given in table 1 . The SE group $(\mathrm{n}=78)$ consisted of 33 men and 45 women with a median age of 59 years. The PG group $(n=71)$ included 28 men and 43 women with a median age of 64 years. The median body mass index for the SE group was 23.9 and 
Table 3. Postoperative data within 30 days

\begin{tabular}{lclc}
\hline & $\begin{array}{l}\text { SE group } \\
(\mathrm{n}=78)\end{array}$ & $\begin{array}{l}\text { PG group } \\
(\mathrm{n}=71)\end{array}$ & $\mathrm{p}$ \\
\hline Total complications & $26(33 \%)$ & $9(13 \%)$ & 0.003 \\
$\quad$ Intra-abdominal infections $\quad 8(10 \%)$ & $2(3 \%)$ & 0.070 \\
$\quad$ Anastomotic leakages & $4(5 \%)$ & $1(1 \%)$ & 0.369 \\
$\quad$ Extra-abdominal infections & $11(14 \%)$ & $4(6 \%)$ & 0.086 \\
$\quad$ Wound infections & $7(9 \%)$ & $1(1 \%)$ & 0.041 \\
$\quad$ Noninfectious complications & $7(9 \%)$ & $3(4 \%)$ & 0.247 \\
Reoperations due to anastomotic leakage & $4(5 \%)$ & $1(1 \%)$ & 0.369 \\
Secondary surgery & $7(9 \%)$ & $5(7 \%)$ & 0.665 \\
Mortality & $3(4 \%)$ & $2(3 \%)$ & 0.728 \\
Median hospital stay, days (interquartile range) & $8(6-12)$ & $8(6-12)$ & 0.533 \\
\hline
\end{tabular}

24.0 for the PG group. The two groups were comparable for type and number of procedures performed (table 2). No differences were seen in median operating time or blood loss.

The postoperative complications are shown in table 3 . The overall postoperative morbidity was significantly higher in the SE group (26 vs. 9, p = 0.003). Specifically, the incidence of wound infections was significantly higher in the SE group (7 vs. $1, \mathrm{p}=0.041$ ). Furthermore, a trend was observed in the incidence of intra-abdominal infections which was higher in the SE group ( 8 vs. $2, \mathrm{p}=$ 0.070). Also a trend toward more extra-abdominal infectious complications was seen in the SE group (11 vs. 4, $\mathrm{p}=0.086)$.

No significant difference was seen in the incidence of anastomotic leakages (4 vs. $1 \mathrm{SE}$ vs. PG group; $\mathrm{p}=0.369$ ). All patients with anastomotic dehiscence required reoperation. No mortality due to anastomotic leakage was observed. No difference was seen in hospital stay between both groups. The mortality was similar for both groups and was based on myocardial pathology in all patients.

\section{Discussion}

Mechanical bowel preparation has been standard protocol in gastrointestinal surgery for more then a century. Together with prophylactic antibiotics and advances in surgical techniques, it is considered to be of high importance in reducing complications in colorectal surgery. Unfortunately, mechanical cleaning with PEG does not always result in a completely clean colon. Zmora et. al. [3] reported fluid or semifluid stool in the colon after what was considered a thorough cleaning. Fluid stool is more difficult to control during surgery and may, therefore, re- sult in higher intraoperative spillage rates and in more complications.

Hughes [4] was one of the first to question the necessity of bowel preparation after a small randomized trial. However, many more randomized trials were necessary before the surgical dogma of bowel preparation was even open for discussion [5-7]. In The Netherlands, a large multicenter trial was started which included 1,433 patients [1]. The incidence of anastomotic leakages was comparable in both groups, approximately $5 \%$. No differences were seen in postoperative morbidity or mortality. After these results were presented, many hospitals changed their protocols. At the beginning of 2005, our clinic changed from mechanical bowel preparation using polyethylene glycol to a single preoperative enema. However, even though there is sufficient evidence in the literature to support this change, a lot of opposition was met in our clinic. We, therefore, planned an evaluation after 1 year. The current study shows that there was no difference in the incidence of anastomotic leakages. There were, however, significantly more wound infections in the patients without mechanical bowel preparation. Furthermore, there was a trend toward more intra- and extra-abdominal infectious complications in patients who had a single enema. These results are partly in contrast to the Cochrane data [2], where no convincing evidence was found that bowel preparation would reduce the incidence of infections.

These data are not the first which show negative results of the introduction of a single preoperative enema. Platell et al. [8] in a randomized study with 300 patients showed that even though there was no significant difference between the two groups in overall anastomotic leaks, there was a significant difference in the severity of the leaks. In this study, 4 patients who had an enema devel- 
oped an anastomotic leak; in all of these cases reoperation was required. One patient in the PEG group developed an anastomotic leak, also requiring a reoperation. In this study, nearly $30 \%$ of the patients had a low anastomosis, known for a higher leakage rate.

The results of this study caused a new discussion in our clinic: should we restart mechanical bowel preparation or not? An important factor in this discussion was our experience that laparoscopic colonic surgery was sometimes technically more demanding due to a large fecal load which made the colon difficult to handle.

Based on both the conflicting data currently available from different randomized studies [1-8] and our results, the preoperative bowel preparation protocol in our institution has been changed. All Patients with an indication for a left-sided colonic resection are routinely prepared with polyethylene glycol, unless a contraindication for mechanical bowel preparation exists.

\section{References}

1 Contant CM, Sant van't H, Hop WC, Oostvogel HJ, Weidema WF: POCON-Studie: Preoperatieve Orale Darmvoorbereiding Onzin of Niet? Nederlandse Vereniging voor Heelkunde 2005; Abstract V02.01.

2 Guenaga KF, Matos D, Castro AA, Atallah AN, Wille-Jorgensen P: Mechanical bowel preparation for elective colorectal surgery. Cochrane Database Syst Rev 2005: CD001544.

3 Zmora O, Mahajna A, Bar-Zakai B, Rosin D, Hershko D, Shabtai M, Krausz MM, Ayalon A: Colon and rectal surgery without mechanical bowel preparation: a randomized prospective trial. Ann Surg 2003;237:363-367.

\footnotetext{
Hughes ES: Asepsis in large-bowel surgery Ann R Coll Surg Engl 1972;51:347-356.

$\checkmark 5$ Burke P, Mealy K, Gillen P, Joyce W, Traynor O, Hyland J: Requirement for bowel preparation in colorectal surgery. Br J Surg 1994;81: 907-910.

-6 Santos JC, Batista J, Sirimarco MT, Guimaraes AS, Levy CE: Prospective randomized trial of mechanical bowel preparation in patients undergoing elective colorectal surgery. Br J Surg 1994;81:1673-1676.
}

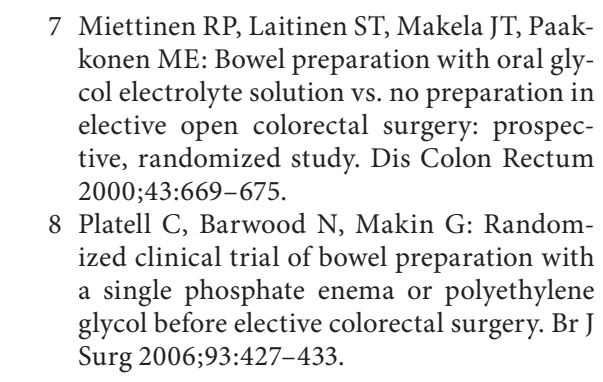

\section{Invited Commentary}

\section{S. Galandiuk}

Louisville, Ky., USA

The authors present a fascinating report of what happens when one changes one's clinical practices based upon results of a randomized prospective clinical trial and then finds that these practices are associated with increased morbidity! As the authors recount, at the beginning of 2004, a Dutch randomized study comparing preoperative mechanical bowel preparation to a single preoperative enema prior to elective bowel resection reported no differences in anastomotic leak rate, septic complications, or mortality. On the basis of that study, the authors changed their clinical practice in their own institution. When they did this, they, however, wisely continued to study their patients and planned to reevaluate these practices at the end of 1 year. When they performed their reassessment, they found, to their surprise, that the overall postoperative morbidity and wound infection rates were both higher in the enema group in addition to clinically, but not statistically, higher rates of intra-abdominal and extra-abdominal infectious complications.

Notwithstanding the small patient numbers and the fact that this patient series is consecutive, this really represents the very best of surgical science, i.e., surgeons paying attention in taking care of their patients. I personally know of several other surgeons who have tried to emulate the 'no bowel preparation' example and also noted clinically higher complication rates.

In performing clinical studies, many variables and biases may come into play. Even in meta-analyses, biases of individual studies can bias the outcome of the analysis as a whole. It is important not to forget that a clinician who has the patient's interest paramount must independently evaluate clinical practices and decide whether they are 
right for his or her patients and his or her institution. I commend the authors on their attention to detail, their scientific inquiry, and their continuing assessment of their results even after the randomized study was completed. While the jury is still out on the question of pre- operative bowel preparation, these authors and their patients will continue to benefit from their careful attention to detail. We can only hope that surgeons and surgical trainees will continue to put their patients and their patients' interests first in this manner.

\title{
Invited Commentary
}

\author{
Hein G. Gooszen
}

Utrecht, The Netherlands

The authors have addressed the issue of how to optimally prepare the colon to reduce the number of those complications that are directly attributable to no or inadequate large bowel preparation in elective large-bowel surgery. A plethora of studies have been published in the last decade, and the question remaining is: Can patients, who need a low anterior resection for cancer, with or without a temporary defunctioning ileostomy, be electively operated without any form of large-bowel preparation?

These patients are at risk of anastomotic leakage because of the site of the anastomosis as such with an imminent higher risk for leakage and because of the reduced quality of anastomotic healing as a result of the preoperative irradiation most of them needed.

Their risk of anastomotic leakage is fundamentally different from the risk in right hemicolectomy, transversectomy, and left hemicolectomy for cancer without preoperative irradiation or ileocoecal resection for Crohn's disease. The authors focus in their conclusion on 'elective left-sided large-bowel surgery', but in their material about $35 \%$ have undergone other types of resection with a lower risk for leakage.

In surgery for benign disease, like diverticular disease, many surgeons do not hesitate to perform a primary anastomosis in an unprepared bowel even in acute cases, if not in the very sick, frail, and elderly patient with comorbidity like chronic obstructive pulmonary disease, coronary heart disease, uraemia, or diabetes.

This, however, is largely based on level II, III, and IV evidence as the best available. Randomized controlled trials on treatment of perforated diverticular disease have been attempted but failed. It is unlikely that such a trial will ever be conducted given the fact that in the young and otherwise fit patient the risk of leakage of a primary anastomosis is acceptable and that in the frail and elderly an anastomotic leakage may be a lethal complication.
With all these considerations in mind and given the fact that the optimal preparation of a low left-sided colonic anastomosis is the remaining question to be answered, this study does not really add to the discussion.

In the description of the complications that have added to the conclusions drawn at the end, the complications are not ascribed to the type of operation responsible for the complication observed. It can be anticipated that even more than half of the patients had benign disease with a different risk profile from that of patients who had an anterior resection, most likely for cancer.

Consequently, it is not clear which patients had intraabdominal infections who had anastomotic leakage, and whether or not another regime of bowel preparation could have theoretically prevented this.

The subgroups that really matter, because they are the heart of the discussion and the conclusion, are the 6 patients in the single-enema group and the 10 polyethylene glycol group patients. Put together with the 50 patients that needed sigmoid resection, the reader is still only left with $<50 \%$ of the patients to base the final conclusion on.

These groups are too small for a statistically valid conclusion in a nonrandomized comparative study, maybe even if the four anastomotic leakages that occurred in the single-enema group would have been observed in the 'anterior resection' subgroup. If the change in the protocol by the Amsterdam group would have been based on the results they found, their conclusion is seriously flawed.

The major reason for going back to polyethylene glycol preparation of the large bowel is the group's preference for an empty colon to be removed laparoscopically. The group has pioneers in the field of laparoscopic surgery and a reputation to defend. Their preference is understandable, but based on inconclusive literature and personal preference and not on the data they have presented. 